\title{
ÉTUDE ET RÉALISATION D'UNE BOBINE DE STOCKAGE D'ÉNERGIE MAGNÉTIQUE ADAPTÉE A UNE DYNAMO UNIPOLAIRE IMPULSIONNELLE DE 5 MJ
}

\author{
B. GEOFFRION et M. LEGENTIL
}

\author{
Laboratoire de Physique des Plasmas, Groupe Fusion, Université Paris-Sud 91405 Orsay, France
}

(Reçu le 12 juin 1974)

\begin{abstract}
Résumé. - La présente étude s'inscrit dans un programme de réalisation de générateurs impulsionnels de grande puissance, dont l'élément de base est une dynamo homopolaire, sans fer, précédemment décrite [1]. Nous déterminons, ici, le deuxième élément constitué par une bobine magnétique, chargée par le courant de la génératrice. Nous donnons, ensuite, les résultats expérimentaux obtenus avec l'ensemble réalisé.

L'énergie maximale stockée est de 1,5 MJ, sous la forme d'un champ magnétique de l'ordre de $2,5 \mathrm{~T}$, dans un volume d'air voisin du mètre cube. La constante de temps de l'ensemble est de $0,4 \mathrm{~s}$.

Abstract. - The present study is part of a construction programme, for high power pulse generators for which the basic energy source is an iron-free self-excited homopolar generator [1]. We describe here an energy storage coil, and we discuss the experimental results obtained for the units which we have constructed.

The maximum energy stocked is $1.5 \mathrm{MJ}$, magnetic field of 2.5 tesla in one cubic metre, the total time constant being $0,4 \mathrm{~s}$.
\end{abstract}

1. Introduction. - En vue d'expérience de physique sur des plasmas à haute densité, nous cherchons à réaliser une source d'énergie de grande puissance. L'élément de base est une dynamo homopolaire impulsionnelle sans fer de grande puissance massique semblable à celle décrite précédemment [1] et dont l'énergie cinétique maximum stockée est de $5 \mathrm{MJ}$.

Rappelons que ce type de génératrice est constitué d'un rotor lancé à grande vitesse et d'un circuit électrique autoexcité, sans fer, fermé par injection de mercure. La force électromotrice est faible, de l'ordre de la dizaine de volts, mais l'impulsion de courant obtenue atteint une valeur crête de l'ordre du mégaampère pour une largeur de l'ordre de quelques dizièmes de secondes.

Notre but ultérieur étant d'obtenir des impulsions plus courtes (par exemple de l'ordre de quelques microsecondes) il nous faut transformer l'énergie cinétique initiale du volant de la machine en une forme apte à un raccourcissement des temps d'impulsions utiles, avec évidemment le meilleur rendement possible. Il existe de nombreuses solutions pour l'obtention d'un tel résultat; la plus simple consistant à transférer l'énergie de la machine vers une inductance convenable, puis à dériver l'énergie magnétique ainsi stockée vers la charge à l'aide d'interrupteurs à ouverture très rapide.
Dans tous les procédés envisageables en pratique, il nous faut disposer transitoirement d'énergie inductive : la charge de la génératrice sera donc une bobine de stockage aux caractéristiques un peu particulières ; compte tenu de celles de la machine [1] :

- impédance faible $(\simeq 1 \mu \mathrm{H}, 1 \mu \Omega)$,

- grande constante de temps $(\simeq 1 \mathrm{~s})$.

La bobine aura donc peu de tours.

De plus, la récupération de l'énergie magnétique stockée étant beaucoup plus rapide que la constante de temps, nous pouvons considérer que toute ligne de force située dans les conducteurs sera "gelée " au moment de la transformation : l'énergie du champ magnétique intérieur aux conducteurs est perdue. Le meilleur rendement exige donc une bobine mince.

La génératrice réalisée de $5 \mathrm{MJ}$ avait déjà été expérimentée avec une bobine destinée à produire un champ magnétique intense mais convenant mal au stockage.

Notre problème était donc de réaliser une bobine de stockage adaptée à la génératrice et qui présente des caractéristiques de réalisation convenables, c'est-àdire en particulier une masse de cuivre acceptable.

Nous déterminons, tout d'abord, les caractéristiques de la bobine à construire, puis nous donnons les résultats expérimentaux obtenus avec l'ensemble génératrice-bobine réalisé. 
2. Détermination des caractéristiques de la bobine. Etant donné que nous sommes en régime impulsionnel, il faudra faire intervenir d'une part l'effet de peau et d'autre part une répartition inhomogène possible des courants locaux. L'effet de peau peut être, en première approximation, négligé puisque la bobine est mince. La distribution inhomogène des courants apparaît lorsque ceux-ci ont la possibilité de se répartir pour s'opposer au flux au travers des conducteurs ; cette possibilité dépend de la disposition et du nombre de spires et ne pourra être évaluée qu'une fois la géométrie de la bobine déterminée.

2.1 OPTIMISATION DU RENDEMENT D'UTILISATION POUR UNE BOBINE DE MASSE DONNÉE. — La bobine est définie par quatre paramètres géométriques : sa longueur $l$, son rayon moyen $r$, son épaisseur $e$, son nombre de tours $n$ et par le matériau utilisé dont la résistivité est $\rho$ et la masse volumique $\varpi$.

D'autre part, la bobine est électriquement liée à la génératrice par son inductance totale $\mathcal{L}$ et sa résistance $\mathcal{R}$. Le courant maximum $I_{\mathrm{M}}$ que peut supporter la dynamo étant imposé par la limite du couple mécanique qu'elle subit, l'équation électrique [1] nous donne une relation entre $\mathcal{L}$ et $\mathcal{R}$ :

$$
\left(\mathfrak{L}+\mathfrak{L}_{0}\right) I_{\mathbf{M}}^{2}=2 W_{0}\left(1-\frac{\omega_{\mathrm{c}}}{\omega_{\mathrm{M}}} \frac{\mathcal{R}+\mathcal{R}_{0}}{\mathcal{R}_{0}}\right)^{2}
$$

où $\mathfrak{L}_{0}$ est l'inductance de la machine, $\mathcal{R}_{0}$ sa résistance, $\omega_{\mathrm{c}}$ la vitesse critique en court-circuit et $\omega_{\mathbf{M}}$ la vitesse maximale permise.

Nous pouvons d'autre part calculer en fonction des paramètres géométriques $\mathcal{L}$ et $\mathcal{R}$. La formule des bobines minces [2], suffisante compte tenu des effets négligés et de la précision avec laquelle sont connues les caractéristiques de la génératrice, nous donne $\mathcal{L}$ :

$$
\mathcal{L}=\pi \mu_{0} \frac{n^{2} r^{2}}{l} F\left(\frac{l}{2 r}\right)
$$

où $F$ est une fonction du facteur de forme $l / 2 r$ qui tend vers 1 quand $l / 2 r$ tend vers l'infini. $\mathcal{R}$ est calculée, à répartition de courant homogène $: \mathcal{R}=2 \pi \rho \frac{n r}{e l}$.

Enfin, la masse $M$ de la bobine étant déterminée par d'autres considérations (voir 2.2), nous pouvons écrire une autre relation entre les paramètres géométriques:

$$
M=2 \pi \varpi r e l .
$$

La relation électrique et le choix de la masse réduisent à deux (pour un matériau donné) le nombre de paramètres dont nous disposons : $l$ et $n$. Ce qui nous permet d'écrire le rendement $\eta$ d'utilisation :

$$
\eta=\frac{W_{\mathrm{e}}(l, n)}{W_{0}}=\frac{I_{\mathrm{M}}^{2}}{2 W_{0}}\left(\mathcal{L}-\mathfrak{L}_{\mathrm{i}}\right) .
$$

L'énergie utile est égale à l'énergie totale stockée

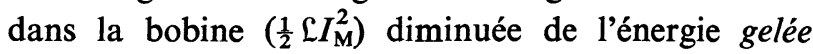

dans les conducteurs $\left(\frac{1}{2} \mathfrak{L}_{\mathrm{i}} I_{\mathrm{M}}^{2}\right)$. Cette dernière est calculée pour une répartition homogène du courant, avec

$$
\mathcal{L}_{\mathrm{i}}=\frac{2 \pi \mu_{0}}{3} \frac{n^{2} r e}{l}
$$

$W_{0}$ est l'énergie cinétique initiale de la machine. Il nous faut alors rechercher les valeurs de $l$ et $n$ qui donnent le rendement maximal. Nous n'expliciterons pas ici les calculs qui ont été publiés par ailleurs [3], nous ne donnons que les résultats obtenus.

La figure 1, montre la variation des valeurs optimales $\eta_{\text {opt }}, l_{\text {opt }}, r_{\text {opt }}$ en fonction de $n$ pour une masse de cuivre allié de $600 \mathrm{~kg}(\rho=2,4 \mu \Omega . \mathrm{cm})$ de bonne

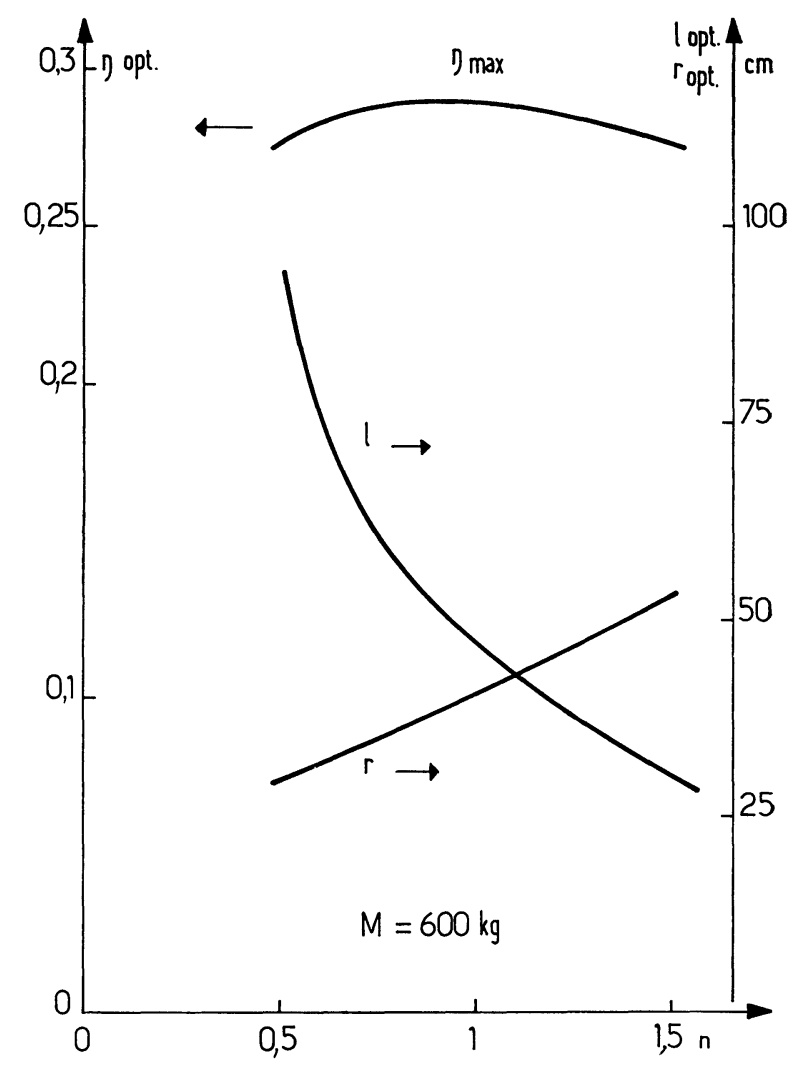

FIG. 1. - Rendement maximum et dimensions optimales en fonction du nombre de tours de la bobine (cuivre allié de résistivité $2,4 \mu \Omega \mathrm{cm}$ ).

tenue mécanique $\left(22 \mathrm{~kg} \mathrm{~mm}^{-2}\right)$. Nous constatons que $\eta_{\text {opt }}$ passe par un maximum assez plat à 0,29 pour $n \sim 0,9$. Nous construirons donc une bobine monospire $\left({ }^{1}\right)$. La valeur de $\mathcal{L}$ varie peu avec $n$ et est de l'ordre de $1 \mu \mathrm{H}$.

2.2 Choix DE LA MASSE. - $\mathrm{Si}$ nous répétons le calcul précédent pour différentes masses, nous obte-

(1) On démontre que le rendement est maximal pour $l / 2 r \sim 0,40$ quelles que soient la génératrice et la masse choisie et $F(l / 2 r)$ est alors voisin de $\frac{1}{2}$. Nous sommes, avec $n=1$ à $l / 2 r=0,45$ donc très voisin de la valeur optimale. Bien que $l / 2 r$ varie vite avec $n . \eta_{\text {opt }}$ est presque constant sur une large plage autour du maximum de $\eta$. 
nons les résultats présentés figure $2, \eta_{\text {Max }}$ croît avec la masse mais de moins en moins vite et le nombre de tours correspondant reste voisin de 1 .

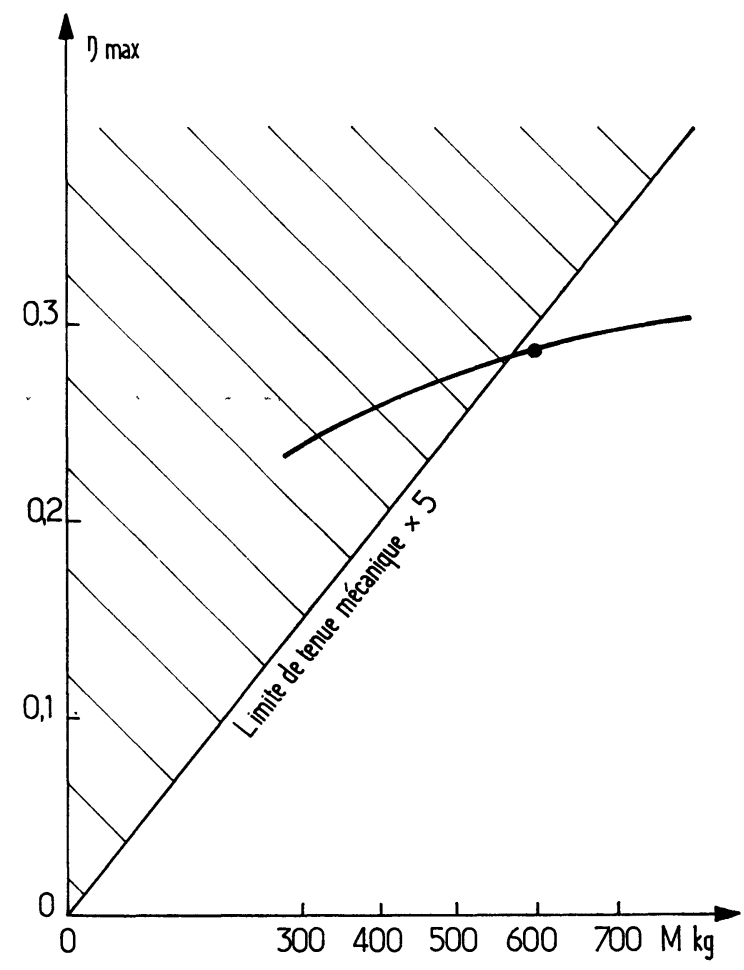

Fig. 2. - Variation du rendement optimum en fonction de la masse de la bobine.

Nous avons donc choisi de construire une bobine monospire par moulage de deux demi-cylindres et de tenir les efforts dus à la pression magnétique par la seule résistance mécanique $\sigma$ du cuivre, c'est-à-dire, que nous devons nous assurer que (cylindre mince soumis à une pression interne) :

$$
\frac{B^{2}}{2 \mu_{0}} \frac{r}{e}<\sigma
$$

en prenant pour valeur maximale de l'induction magnétique :

$$
B=\mu_{0} \frac{n I_{\mathbf{M}}}{l} .
$$

Compte tenu des différentes relations qui lient les caractéristiques de la bobine, nous sommes conduits à adopter une masse de cuivre telle que :

$$
M>\frac{2 \varpi W_{0}}{\sigma} \eta
$$

Soit dans notre cas $M>400 \eta$.

L'utilisation du cuivre moulé exige un fort coefficient de sécurité que nous prenons égal à 5 soit $M=2000 \eta$, droite que nous avons portée sur la figure 2 dont l'intersection avec $\eta_{\text {Max }}(M)$ donne la masse de la bobine : de l'ordre de $600 \mathrm{~kg}$.
Finalement, les caractéristiques de la bobine sont :

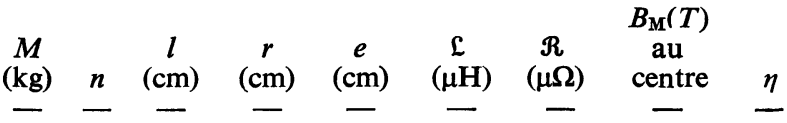

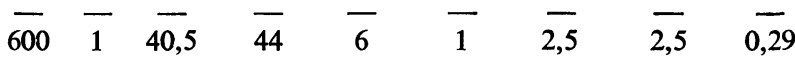

2.3 Calcul Des Distributions SPatiale eT TeMPORELLE DU COURANT. - Il nous reste à calculer les effets d'écran négligés pour connaître leur importance sur les performances et aussi sur les contraintes supplémentaires dues à d'éventuelles densités locales fortes de courant.

Nous avons, d'abord, évalué la distribution radiale de courant, au centre de la bobine en supposant que le courant $I$ débité par la génératrice n'est pas perturbé. Ce courant est de la forme : $I_{M} / \operatorname{ch}(t / \tau)$, [1] $\tau$ étant une constante, que l'on peut développer en $\Sigma I_{\mathrm{K}} \exp \left(\beta_{\mathrm{K}} t\right)$ puis on intègre l'équation de diffusion à travers le conducteur [4]. On trouve que la densité radiale du courant s'uniformise très vite; au courant maximum, l'écart relatif entre densité à la paroi et densité moyenne est de l'ordre de $10 \%$. Ceci est dû au fait que l'épaisseur de la bobine $(6 \mathrm{~cm})$ est de l'ordre de grandeur de la profondeur de pénétration $e_{\mathrm{p}}$ $(\sim 10 \mathrm{~cm})$ pour le temps de montée de la dynamo.

En ce qui concerne la distribution le long d'une génératrice nous ne pouvons plus supposer le courant non perturbé : la bobine étant mince et monospire, le courant va se répartir pour s'opposer à tout flux normal à la paroi, c'est-à-dire se concentrer aux extrémités de la bobine. L'inductance vue par la machine sera plus faible et le courant total plus fort.

On trouve dans la littérature un certain nombre de calculs de distribution de courant dans des bobines cylindriques [par exemple 5] mais dans des cas différents du nôtre (charge par condensateurs, conductivité infinie...).

Pour résoudre ce problème, nous supposons la bobine parfaitement mince et la découpons perpendiculairement à l'axe en $N$ tranches identiques. Nous calculons les mutuelles des spires ainsi obtenues et écrivons leurs équations électriques couplées à celle de la génératrice par la tension et le courant total.

La résolution numérique du système différentiel obtenu [6] montre que l'augmentation du courant total crête par rapport à une distribution homogène est assez faible $(\sim 5 \%)$

La figure 3 montre la variation de ce courant maximal en fonction de la vitesse initiale $\omega$ de la génératrice. Nous avons porté sur la figure 4 la distribution du courant pour différents temps : la densité aux extrémités est, au maximum, égale à 1,5 fois la densité moyenne et à, au moins, 2 fois la densité au centre.

2.4 CAlCul De l'induction magnétiQue. - Nous avons alors calculé de manière précise l'induction magnétique dans la bobine (en tenant compte, ici, de son épaisseur). La répartition du champ est plus uniforme dans l'espace et dans le temps qu'en dis- 


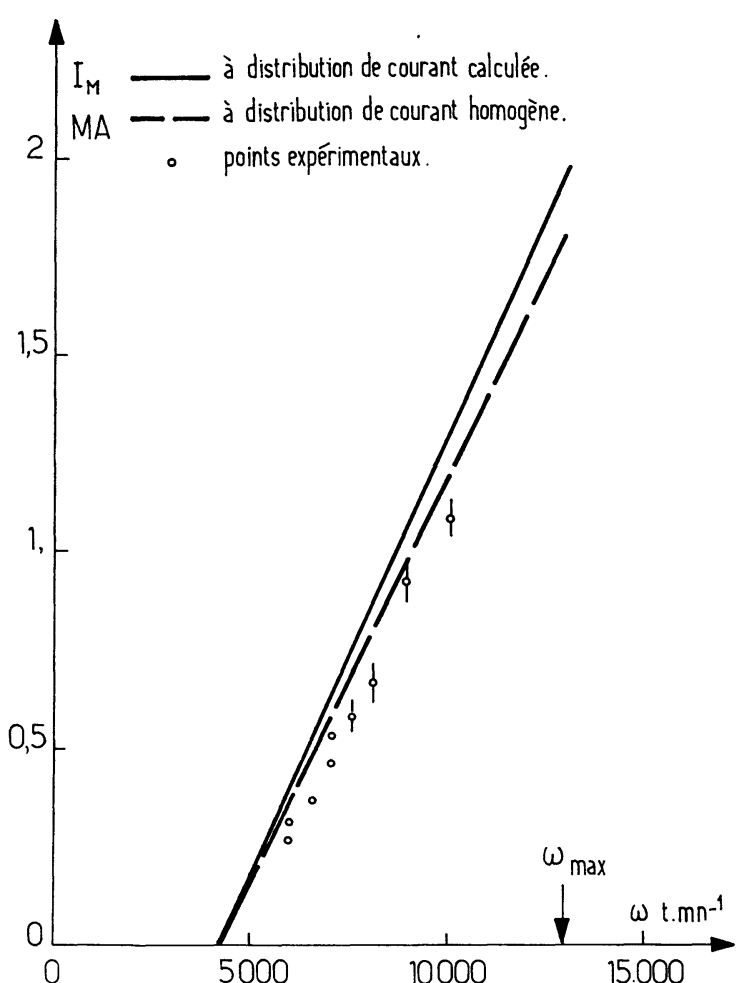

FIG. 3. - Variation du courant maximum en fonction de la vitesse initiale de la génératrice et valeurs expérimentales.

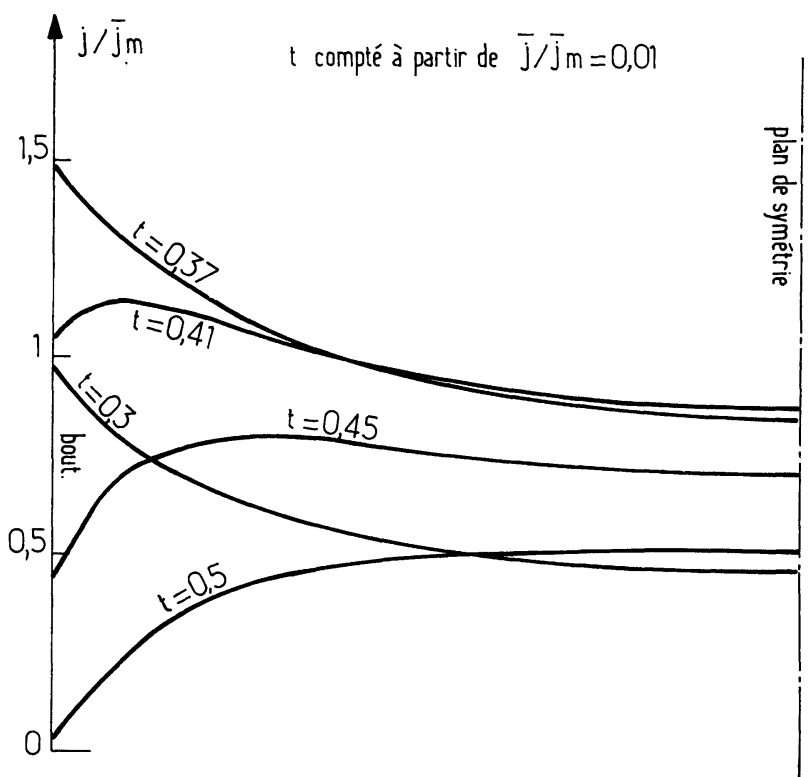

FIG. 4. - Distribution du courant dans la bobine.

tribution homogène de courant, mais les différences sont assez faibles (de l'ordre de 1 à $2 \%$ ), sauf le long de la paroi $(10 \%)$ et aux extrémités pour le champ radial $(20 \%)$.

Du point de vue des efforts radiaux, bien que la densité du courant varie du simple au double, la variation du champ magnétique est insuffisante pour provoquer des contraintes dangereuses.
2.5 Remarque. - Dans l'évaluation du rendement utile, nous avons négligé un phénomène: le champ de réaction dans l'air, qui prendra naissance pendant l'ouverture rapide de la bobine pour assurer la continuité de la composante normale du champ gelé dans les conducteurs. Ce champ sera d'autant plus faible que la composante normale le sera : c'est précisément ce à quoi tend la libre répartition du courant.

3. Etude expérimentale. - 3.1 MOYENS DE MESURE. - La photographie présente l'ensemble génératricebobine schématisé figure 5 .

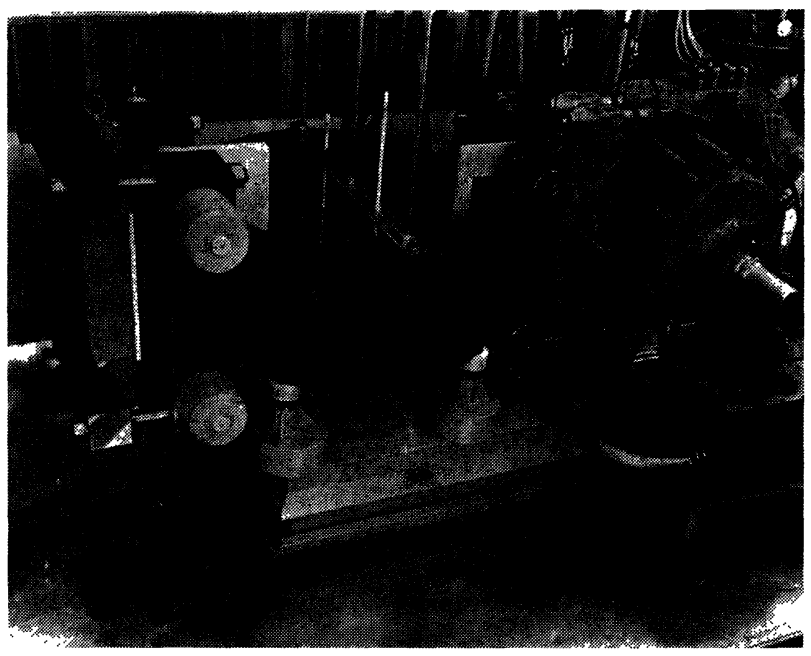

Pното. - La génératrice $5 \mathrm{MJ}$ accouplée à la bobine. On distingue les sondes de champs et de courant.

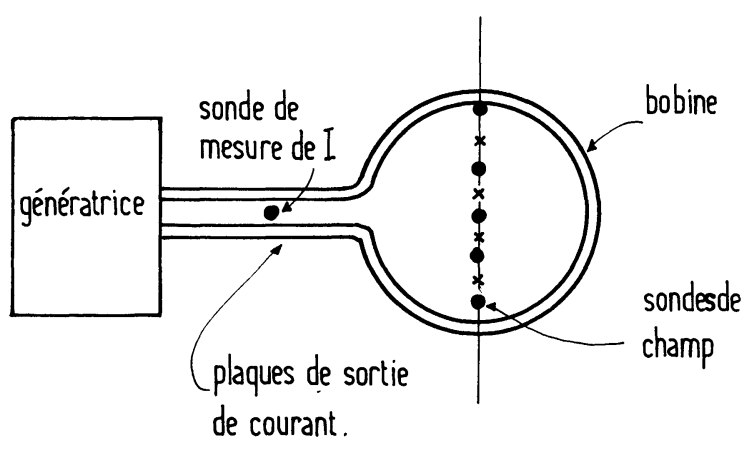

FIG. 5. - Schéma du dispositif expérimental.

Nous avons déterminé la valeur crête du courant $I$ pour différentes vitesses initiales de la dynamo ainsi que les valeurs crête de l'induction magnétique $B$ en différents points du plan médian. Pour un courant crête de 0,55 MA nous avons établi la carte des valeurs crête de l'induction magnétique dans un plan diamétral.

Nous mesurons les dérivées par rapport au temps du courant délivré par la machine et de l'induction magnétique à l'aide de sondes, constituées par des bobinages, dont les caractéristiques dépendent des grandeurs à déterminer (amplitudes, temps). 
Les différents signaux sont envoyés sur des oscillographes cathodiques.

Pour obtenir la valeur crête $I_{\mathrm{M}}$ et $B_{\mathrm{M}}$ nous avons admis la forme théorique :

$$
I=I_{\mathrm{M}} / \operatorname{ch}\left(a I_{\mathrm{M}} t\right)
$$

(pour la bobine, le courant total diffère très peu du courant total obtenu en supposant une distribution homogène 2.3).

La dérivée de $I$ (ou de $B$ ) est donc de la forme :

$$
v=k \frac{\operatorname{th}\left(a I_{\mathrm{M}} t\right)}{\operatorname{ch}\left(a I_{\mathrm{M}} t\right)}
$$

fonction dont les valeurs maximales sont $\pm 0,5 k$; pour $a I_{\mathrm{M}} t= \pm 0,8815$, ce qui nous permet d'obtenir $I_{\mathrm{M}}$ (ou $B_{\mathrm{M}}$ ) par :

$$
\left.I_{\mathrm{M}}^{\mathrm{T}}\left(\text { ou } B_{\mathrm{M}}\right)=k_{I} \text { (ou } k_{B}\right) \frac{\Delta t^{\mathrm{p}} \Delta v}{1,763} \quad \text { (cf. fig. 6) }
$$

On constate que cette méthode donne, aux erreurs expérimentales près, le même résultat que l'intégration numérique.

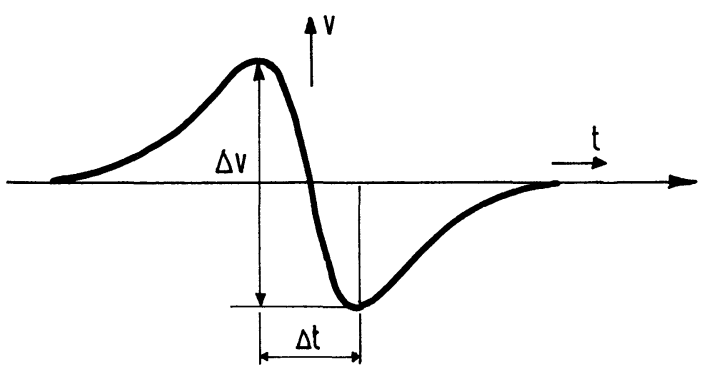

Fig. 6. - Forme de la dérivée en fonction du temps du courant total et grandeurs mesurées.

Compte tenu des erreurs sur $\Delta t, \Delta v$, l'erreur sur $I_{\mathrm{M}}$ (ou $B_{\mathrm{M}}$ ) est en moyenne de l'ordre de $6 \%$. Signalons que nous avons vérifié sur les oscillogrammes la forme en th/ch. Un enregistreur oscillographique à galvanomètre nous a permis de vérifier la forme de la dérivée du courant total avec une bonne précision (Fig. 7).

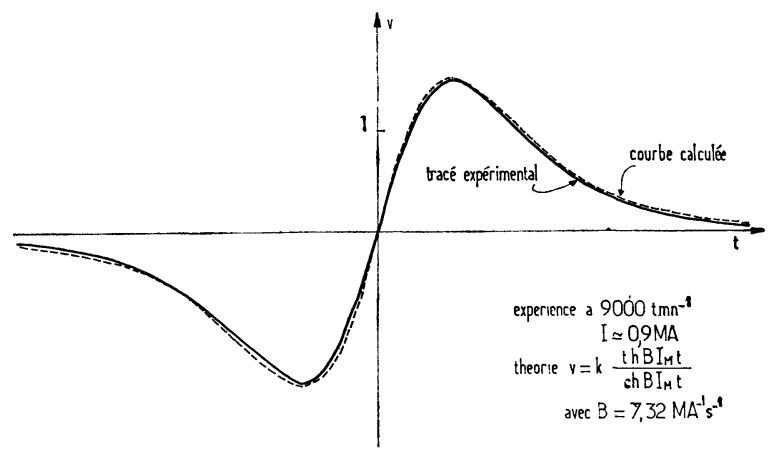

Fig. 7. - Lissage de la dérivée du courant.

REVUE DE PHYSIQUe APPLIQUée. - T. 10, No 1, JANVIER 1975
3.2 RÉSUltats. - Nous avons porté sur la figure 3 la valeur crête du courant en fonction de la vitesse à l'instant initial. Cette vitesse est celle de la machine au début du cycle de fonctionnement pendant lequel le rotor est ralenti par le frottement du mercure et évidemment par le couple électromagnétique.

Le ralentissement dû au mercure est de l'ordre de $1400 \mathrm{tmin}^{-1} \mathrm{~s}^{-1}$ à $10000 \mathrm{tmin}^{-1}$ et modifie la valeur de la vitesse critique [1]. Ceci explique le décalage de la courbe expérimentale par rapport à la courbe théorique.

Nous présentons sur la figure 8 , le courant $I_{\mathrm{M}}$ en fonction de la vitesse à l'instant initial ainsi que la valeur de l'induction magnétique $B_{\mathrm{M}}$ (composante axiale) au centre et près de la paroi intérieure de la bobine (dans le plan médian).

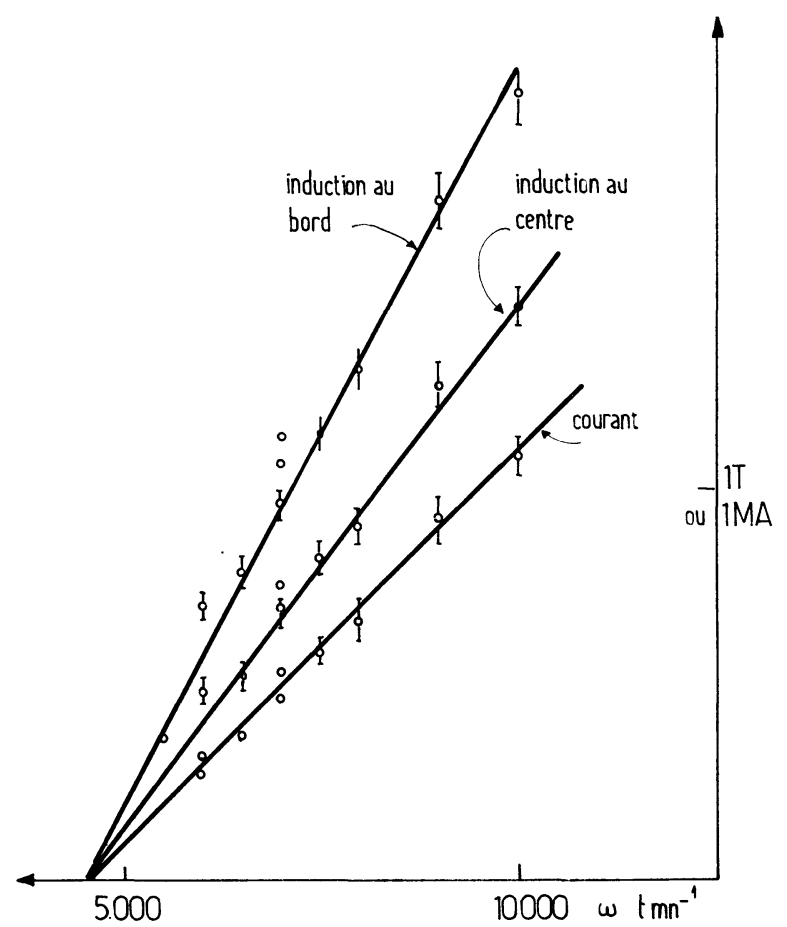

FIG. 8. - Courant et induction dans la bobine en fonction de la vitesse initiale de la génératrice.

Sur la figure 9 sont portées les variations (composante axiale) de l'induction le long de l'axe de la bobine et le long d'une génératrice pour un courant de 0,55 MA : il y a un bon accord pour la valeur sur l'axe. En ce qui concerne les points situés le long de la paroi, le programme ne permet pas le calcul à l'endroit précis où l'on peut placer les sondes, or, l'induction magnétique varie très vite dans ces régions ce qui peut expliquer l'écart existant entre les deux courbes.

La figure 10 donne les valeurs mesurées de l'induction magnétique dans un plan perpendiculaire aux arrivées de courant (Fig. 5) : il s'agit des composantes axiales pour un courant crête de $0,55 \mathrm{MA}$.

Il faut remarquer que les valeurs mesurées du champ 


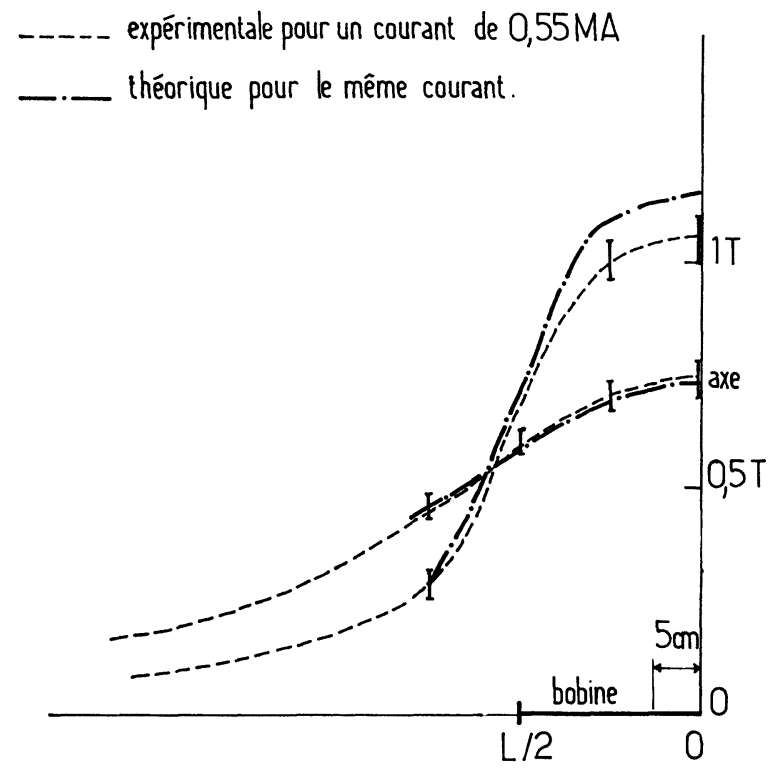

Fig. 9. - Composantes axiales de l'induction magnétique le long de l'axe et d'une génératrice.

ne sont pas symétriques à cause de la présence de matériaux magnétiques dans le voisinage de la partie inférieure de la bobine (cette dernière étant intégrée dans l'ensemble machine-bobine). Seules les valeurs mesurées dans la région vide située au-dessus de la bobine sont donc à comparer aux valeurs théoriques, ce que nous avons admis plus haut. Des impératifs techniques nous ont empêchés de faire les mesures à 1 MA.

4. Intérêts de l'ensemble génératrice bobine réalisé. Les résultats expérimentaux obtenus s'inscrivent dans le cadre de deux orientations de recherche distinctes.

D'une part, des expériences en champ magnétique moyen et quasi stationnaire dans de grands volumes sont de mise en œuvre immédiate. En effet, nous pouvons disposer d'une induction magnétique de l'ordre de 1,5 $\mathrm{T}$ (pour un courant de $1 \mathrm{MA}$ ) dans un volume de 1001 , en admettant un défaut d'homogénéité de $10 \%$. L'induction magnétique est à sa valeur maximale (à $10 \%$ près) pendant $0,15 \mathrm{~s}$. Ce temps augmente si l'on travaille à induction plus faible.

D'autre part, en associant à cet ensemble, des dispositifs disjoncteurs convenables, il est possible de

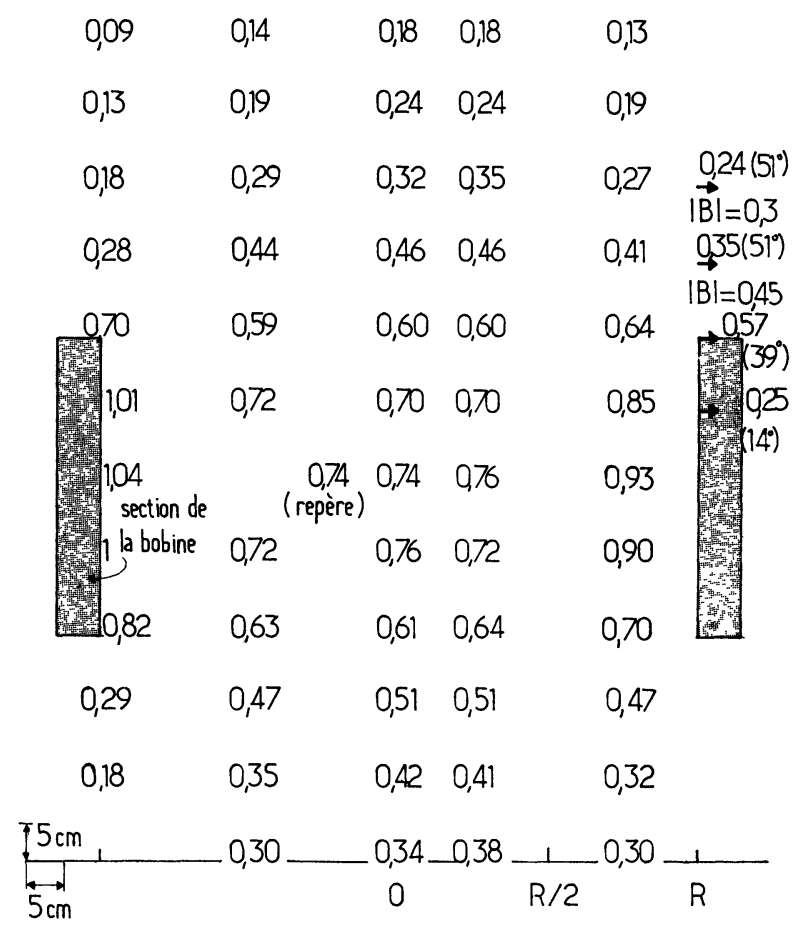

FIG. 10. - Composantes axiales de l'induction pour $I=0,55 \mathrm{MA}$ et quelques composantes radiales. L'angle indiqué est celui de $B$ par rapport à l'axe de la bobine (B est en tesla, $\Delta B / B \sim 5 \%$ ).

réaliser une source d'énergie impulsionnelle très puissante concurrençant avantageusement les grandes batteries de condensateurs ou les générateurs à explosifs par la souplesse de sa mise en œuvre.

Notons également que la réussite de cette réalisation nous a permis d'envisager la construction de générateur à plus haute énergie cinétique initiale (100 MJ) pour lequel le rendement théorique atteint $50 \%$ (ce qui correspond à un champ de $5 \mathrm{~T}$ stockés dans un volume de quelques mètres cubes).

5. Conclusion. - Nous avons élaboré un générateur d'énergie magnétique, impulsionnel, au niveau du MJ, dont les performances sont conformes aux calculs théoriques et nous permettent d'envisager outre la construction d'ensembles plus puissants à plus haut rendement, de multiples expériences en champs forts quasi stationnaires.

\section{Références}

[1] Hahn, R., Lucidarme, J., Rioux, C. et Guillet, R., Revue Phys. Appli 8 (1973) 53. Réalisation et expérimentation d'une génératrice unipolaire impulsionnelle sans fer de très grande puissance massique.

[2] Durand, E., Magnétostatique (Masson) 1968, p. 193.

[3] Legentil, M., Etude d'une bobine de stockage et transfert d'énergie accouplée à une génératrice homopolaire de 100 MJ. Note technique du Laboratoire de Physique des Plasmas Orsay no 1 , LP 81.
[4] Legentil M., Effet de peau lié à la montée du courant dans des bobines de stockage accouplées à une génératrice homopolaire, Note technique du Laboratoire de Physique des Plasmas Orsay $n^{\circ} 4$.

[5] ELLIS, R. E., UCRL 7864 mai 1964.

[6] LegentIL, M., Evolution et distribution du courant et des champs dans les bobines accouplées aux génératrices homopolaires. Note technique du Laboratoire de Physique des Plasmas à Orsay $n^{\circ} 10$. 